\title{
Certain relationships among polygamma functions, Riemann zeta function and generalized zeta function
}

\author{
Junesang Choi ${ }^{1 *}$ and Chao-Ping Chen ${ }^{2}$
}

\section{"Correspondence:}

junesang@mail.dongguk.ac.kr 'Department of Mathematics, Dongguk University, Gyeongju, 780-714, Republic of Korea Full list of author information is available at the end of the article

\begin{abstract}
Many useful and interesting properties, identities, and relations for the Riemann zeta function $\zeta(s)$ and the Hurwitz zeta function $\zeta(s, a)$ have been developed. Here, we aim at giving certain (presumably) new and (potentially) useful relationships among polygamma functions, Riemann zeta function, and generalized zeta function by modifying Chen's method. We also present a double inequality approximating $\zeta(2 r+1)$ by a more rapidly convergent series.
\end{abstract}

MSC: Primary 11M06; 33B15; secondary 40A05; 26D07

Keywords: Riemann zeta function; Hurwitz zeta function; gamma function; psi-function; polygamma functions; Bernoulli numbers; multiple Hurwitz zeta function; Stirling numbers of the first kind; asymptotic formulas

\section{Introduction}

The Riemann zeta function $\zeta(s)$ is defined by

$$
\zeta(s):=\sum_{n=1}^{\infty} \frac{1}{n^{s}} \quad(\Re(s)>1) .
$$

The Hurwitz (or generalized) zeta function $\zeta(s, a)$ is defined by

$$
\zeta(s, a):=\sum_{k=0}^{\infty}(k+a)^{-s} \quad\left(\Re(s)>1 ; a \in \mathbb{C} \backslash \mathbb{Z}_{0}^{-}\right),
$$

where $\mathbb{C}$ and $\mathbb{Z}_{0}^{-}$denote the sets of complex numbers and nonpositive integers, respectively. It is easy to see from the definitions (1.1) and (1.2) that

$$
\zeta(s)=\zeta(s, 1)=\left(2^{s}-1\right)^{-1} \zeta\left(s, \frac{1}{2}\right)=1+\zeta(s, 2) .
$$

It is noted that, in many different ways, the Riemann zeta function $\zeta(s)$ and the Hurwitz zeta function $\zeta(s, a)$ can be continued meromorphically to the whole complex $s$-plane having simple poles only at $s=1$ with their respective residues 1 at this point.

\section{Springer}

(c) 2013 Choi and Chen; licensee Springer. This is an Open Access article distributed under the terms of the Creative Commons Attribution License (http://creativecommons.org/licenses/by/2.0), which permits unrestricted use, distribution, and reproduction in any medium, provided the original work is properly cited. 
The polygamma functions $\psi^{(n)}(s)(n \in \mathbb{N})$ are defined by

$$
\psi^{(n)}(s):=\frac{\mathrm{d}^{n+1}}{\mathrm{~d} z^{n+1}} \log \Gamma(s)=\frac{\mathrm{d}^{n}}{\mathrm{~d} s^{n}} \psi(s) \quad\left(n \in \mathbb{N}_{0} ; s \in \mathbb{C} \backslash \mathbb{Z}_{0}^{-}\right)
$$

where $\mathbb{N}$ denotes the set of positive integers and $\mathbb{N}_{0}:=\mathbb{N} \cup\{0\}$, and $\Gamma(s)$ is the familiar gamma function and psi (or digamma) function $\psi$ is defined by

$$
\psi(s):=\frac{\mathrm{d}}{\mathrm{d} s} \log \Gamma(s) \quad \text { and } \quad \psi^{(0)}(s)=\psi(s)
$$

Here is a well-known (useful) relationship between the polygamma functions $\psi^{(n)}(s)$ and the generalized zeta function $\zeta(s, a)$ :

$$
\begin{gathered}
\psi^{(n)}(s)=(-1)^{n+1} n ! \sum_{k=0}^{\infty} \frac{1}{(k+s)^{n+1}}=(-1)^{n+1} n ! \zeta(n+1, s) \\
\left(n \in \mathbb{N} ; s \in \mathbb{C} \backslash \mathbb{Z}_{0}^{-}\right) .
\end{gathered}
$$

It is also easy to have the following expression (cf. [1, Eq. 1.2(54)] and [2, Eq. 1.3(54)]):

$$
\psi^{(n)}(s+m)=\psi^{(n)}(s)+(-1)^{n} n ! \sum_{k=1}^{m} \frac{1}{(s+k-1)^{n+1}} \quad\left(m, n \in \mathbb{N}_{0}\right),
$$

which, in view of (1.6), can be expressed in the following form:

$$
\zeta(n, s+m)=\zeta(n, s)-\sum_{k=1}^{m} \frac{1}{(s+k-1)^{n}} \quad\left(n \in \mathbb{N} \backslash\{1\} ; m \in \mathbb{N}_{0}\right),
$$

where an empty sum is (elsewhere throughout this paper) understood to be nil.

The Riemann zeta function $\zeta(s)$ in (1.1) plays a central role in the applications of complex analysis to number theory. The number-theoretic properties of $\zeta(s)$ are exhibited by the following result known as Euler's formula, which gives a relationship between the set of primes and the set of positive integers:

$$
\zeta(s)=\prod_{p}\left(1-p^{-s}\right)^{-1} \quad(\Re(s)>1)
$$

where the product is taken over all primes. It is readily seen that $\zeta(s) \neq 0(\Re(s)=\sigma \geqq 1)$, and the Riemann's functional equation for $\zeta(s)$

$$
\zeta(s)=2(2 \pi)^{s-1} \Gamma(1-s) \sin \left(\frac{1}{2} \pi s\right) \zeta(1-s)
$$

shows that $\zeta(s) \neq 0(\sigma \leqq 0)$ except for the trivial zeros in

$$
\zeta(-2 n)=0 \quad(n \in \mathbb{N})
$$


Furthermore, in view of the following known relation:

$$
\zeta(s)=\frac{1}{1-2^{1-s}} \sum_{n=1}^{\infty} \frac{(-1)^{n-1}}{n^{s}} \quad(\Re(s)>0 ; s \neq 1)
$$

we find that $\zeta(s)<0(s \in \mathbb{R} ; 0<s<1)$. The assertion that all the non-trivial zeros of $\zeta(s)$ have real part $\frac{1}{2}$ is popularly known as the Riemann hypothesis which was conjectured (but not proven) in the memoir of Riemann [3]. This hypothesis is still one of the most challenging mathematical problems today (see Edwards [4]), which was unanimously chosen to be one of the seven greatest unsolved mathematical puzzles of our time, the so-called millennium problems (see Devlin [5]).

Leonhard Euler (1707-1783), in 1735, computed the Basel problem:

$$
1+\frac{1}{4}+\frac{1}{9}+\frac{1}{16}+\frac{1}{25}+\cdots=\zeta(2)=\frac{\pi^{2}}{6}
$$

to 20 decimal places with only a few terms of his powerful summation formula discovered in the early 1730s, now called the Euler-Maclaurin summation formula. This probably convinced him that the sum in (1.13) equals $\pi^{2} / 6$, which he proved in the same year 1735 (see [6]). Euler also proved

$$
\zeta(2 n)=(-1)^{n+1} \frac{(2 \pi)^{2 n}}{2(2 n) !} B_{2 n} \quad\left(n \in \mathbb{N}_{0}\right),
$$

where $B_{n}$ are the Bernoulli numbers (see [1, Section 1.6]; see also [2, Section 1.7]). Subsequently, many authors have proved the Basel problem (1.13) and Eq. (1.14) in various ways (see, e.g., [7]).

We get no information about $\zeta(2 n+1)(n \in \mathbb{N})$ from Riemann's functional equation, since both members of (1.10) vanish upon setting $s=2 n+1(n \in \mathbb{N})$. In fact, until now no simple formula analogous to (1.14) is known for $\zeta(2 n+1)$ or even for any special case such as $\zeta(3)$. It is not even known whether $\zeta(2 n+1)$ is rational or irrational, except that the irrationality of $\zeta$ (3) was proved recently by Apéry [8]. But it is known that there are infinitely many $\zeta(2 n+1)$ which are irrational (see [9] and [10]).

The following formulae involving $\zeta(2 k+1)$ were given by Ramanujan (see [11]):

(i) If $k \in \mathbb{N} \backslash\{1\}$,

$$
\alpha^{k}\left[\frac{1}{2} \zeta(1-2 k)+\sum_{n=1}^{\infty} \frac{n^{2 k-1}}{e^{2 n \alpha}-1}\right]=(-\beta)^{k}\left[\frac{1}{2} \zeta(1-2 k)+\sum_{n=1}^{\infty} \frac{n^{2 k-1}}{e^{2 n \beta}-1}\right] ;
$$

(ii) If $k \in \mathbb{N}$,

$$
\begin{aligned}
0= & \frac{1}{(4 \alpha)^{k}}\left[\frac{1}{2} \zeta(2 k+1)+\sum_{n=1}^{\infty} \frac{1}{n^{2 k+1}\left(e^{2 n \alpha}-1\right)}\right] \\
& -\frac{1}{(-4 \beta)^{k}}\left[\frac{1}{2} \zeta(2 k+1)+\sum_{n=1}^{\infty} \frac{1}{n^{2 k+1}\left(e^{2 n \beta}-1\right)}\right] \\
& +\sum_{j=0}^{\left[\frac{k+1}{2}\right]} \frac{(-1)^{j} \pi^{2 j} B_{2 j} B_{2 k-2 j+2}}{(2 j) !(2 k-2 j+2) !}\left[\alpha^{k-2 j+1}+(-\beta)^{k-2 j+1}\right],
\end{aligned}
$$


where $B_{j}$ is the $j$ th Bernoulli number, $\alpha>0$ and $\beta>0$ satisfy $\alpha \beta=\pi^{2}$, and $\sum^{\prime}$ means that when $k$ is an odd number $2 m-1$, the last term of the right-hand side in (1.16) is taken as $\frac{(-1)^{m} \pi^{2 m} B_{2 m}^{2}}{(m !)^{2}}$.

In 1928, Hardy [12] proved (1.15). In 1970, Grosswald [13] proved (1.16). In 1970, Grosswald [14] gave another expression of $\zeta(2 k+1)$. In 1983, Zhang [11] not only proved Ramanujan formulae (1.15) and (1.16), but also gave an explicit expression of $\zeta(2 k+1)$. For various series representations for $\zeta(2 n+1)$, see [15] and also see [1, Chapter 4] and [2, Chapter 4].

Many useful and interesting properties, identities, and relations for $\zeta(s)$ and $\zeta(s, a)$ have been developed, for example, the formulas recalled above. Here, we aim at presenting certain (presumably) new and (potentially) useful relationships among polygamma functions, Riemann zeta function, and generalized zeta function by mainly modifying Chen's method [16]. We also give a double inequality approximating $\zeta(2 r+1)(r \in \mathbb{N})$ by a more rapidly convergent series, that is, the Dirichlet lambda function $\lambda(s)$ defined by

$$
\lambda(s)=\sum_{k=1}^{\infty} \frac{1}{(2 k-1)^{s}} \quad(\Re(s)>1)
$$

(see Theorem 3).

\section{Main results}

We first prove a relationship between polygamma functions and Riemann zeta functions asserted by Theorem 1 .

Theorem 1 For each $r \in \mathbb{N}$, the following formula holds true:

$$
\begin{aligned}
\frac{1}{(2 r) !} \sum_{j=1}^{n} \psi^{(2 r)}(j+1)= & \zeta(2 r+1)+\frac{1}{(2 r) !}(n+1) \psi^{(2 r)}(n+2) \\
& +\frac{1}{(2 r-1) !} \psi^{(2 r-1)}(n+2)-\zeta(2 r) \quad(n \in \mathbb{N})
\end{aligned}
$$

Proof We prove Eq. (2.1) by using the principle of mathematical induction on $n \in \mathbb{N}$. For $n=1$, in view of Eq. (1.6), it is found that both sides of (2.1) equal $1-\zeta(2 r+1)$. Assume that (2.1) holds true for some $n \in \mathbb{N}$. Then we will show that (2.1) is true for $n+1$, i.e.,

$$
\begin{aligned}
\frac{1}{(2 r) !} \sum_{j=1}^{n+1} \psi^{(2 r)}(j+1)= & \zeta(2 r+1)+\frac{1}{(2 r) !}(n+2) \psi^{(2 r)}(n+3) \\
& +\frac{1}{(2 r-1) !} \psi^{(2 r-1)}(n+3)-\zeta(2 r) .
\end{aligned}
$$

Let denote the left-hand side of (2.2) by $\mathcal{L}$. Then, by the induction hypothesis, we have

$$
\begin{aligned}
\mathcal{L} & =\frac{1}{(2 r) !} \sum_{j=1}^{n} \psi^{(2 r)}(j+1)+\frac{1}{(2 r) !} \psi^{(2 r)}(n+2) \\
& =\zeta(2 r+1)+\frac{1}{(2 r) !}(n+1) \psi^{(2 r)}(n+2)+\frac{1}{(2 r-1) !} \psi^{(2 r-1)}(n+2)-\zeta(2 r)
\end{aligned}
$$




$$
\begin{aligned}
& +\frac{1}{(2 r) !} \psi^{(2 r)}(n+2) \\
= & \zeta(2 r+1)+\frac{1}{(2 r) !}(n+2) \psi^{(2 r)}(n+2)+\frac{1}{(2 r-1) !} \psi^{(2 r-1)}(n+2)-\zeta(2 r) .
\end{aligned}
$$

By using (1.7), we find

$$
\begin{aligned}
\mathcal{L}= & \zeta(2 r+1)+\frac{1}{(2 r) !}(n+2)\left\{\psi^{(2 r)}(n+3)-\frac{(2 r) !}{(n+2)^{2 r+1}}\right\} \\
& +\frac{1}{(2 r-1) !}\left\{\psi^{(2 r-1)}(n+3)+\frac{(2 r-1) !}{(n+2)^{2 r}}\right\}-\zeta(2 r) \\
= & \zeta(2 r+1)+\frac{1}{(2 r) !}(n+2) \psi^{(2 r)}(n+3)+\frac{1}{(2 r-1) !} \psi^{(2 r-1)}(n+3)-\zeta(2 r),
\end{aligned}
$$

which is equal to the right-hand side of (2.2). Hence, by the principle of mathematical induction, (2.1) is true for all $n \in \mathbb{N}$.

We recall the following asymptotic formula for the polygamma function $\psi^{(n)}(s)$ (see [17, p.260, Entry 6.4.11]):

$$
\begin{gathered}
\psi^{(n)}(s) \sim(-1)^{n+1}\left[\frac{(n-1) !}{s^{n}}+\frac{n !}{2 s^{n+1}}+\sum_{k=1}^{\infty} B_{2 k} \frac{(2 k+n-1) !}{(2 k) ! s^{2 k+n}}\right] \\
(|s| \rightarrow \infty ;|\arg (s)| \leqq \pi-\epsilon(0<\epsilon<\pi) ; n \in \mathbb{N}) .
\end{gathered}
$$

Next we give an interesting series representation for $\zeta(2 r)$ in terms of the generalized zeta functions $\zeta(2 r+1, j+1)\left(j \in \mathbb{N}_{0}\right)$.

Theorem 2 The following formula holds true:

$$
\zeta(2 r)=\sum_{j=0}^{\infty} \zeta(2 r+1, j+1) \quad(r \in \mathbb{N}) .
$$

Proof Applying the series representation (1.6) for $\psi^{(n)}(s)$ to the left-hand side of (2.1), we have

$$
\begin{aligned}
-\sum_{j=1}^{n} \sum_{\ell=0}^{\infty} \frac{1}{(\ell+j+1)^{2 r+1}=} & \zeta(2 r+1)-\zeta(2 r) \\
& +\frac{1}{(2 r) !}(n+1) \psi^{(2 r)}(n+2)+\frac{1}{(2 r-1) !} \psi^{(2 r-1)}(n+2) .
\end{aligned}
$$

Taking the limit on each side of the last resulting identity as $n \rightarrow \infty$, in view of (2.3), we obtain the following identity:

$$
\zeta(2 r)=\zeta(2 r+1)+\sum_{j=1}^{\infty} \sum_{\ell=1}^{\infty} \frac{1}{(\ell+j)^{2 r+1}} \quad(r \in \mathbb{N}) .
$$

Now it is easy to find from (1.2) and (1.3) that (2.5) equals (2.4). 
We present a relationship between $\zeta(2 r+1)$ and $\psi^{(2 r)}\left(n+\frac{1}{2}\right)$ asserted by the following theorem.

Lemma For each $r \in \mathbb{N}$, the following formula holds true:

$$
\begin{aligned}
\sum_{k=1}^{n} \frac{1}{(2 k-1)^{2 r+1}}= & \left(1-\frac{1}{2^{2 r+1}}\right) \zeta(2 r+1) \\
& +\frac{1}{2^{2 r+1}(2 r) !} \psi^{(2 r)}\left(n+\frac{1}{2}\right) \quad(n \in \mathbb{N}) .
\end{aligned}
$$

Proof We prove Eq. (2.6) by using the principle of mathematical induction on $n \in \mathbb{N}$. When $n=1$, the left-hand side of (2.6) is clearly 1 , and the right-hand side of (2.6) is seen to be 1 by using (1.6) and (1.3). Assume that (2.6) holds true for some $n \in \mathbb{N}$. Then, by the induction hypothesis and using (1.7), we find

$$
\begin{aligned}
\sum_{k=1}^{n+1} \frac{1}{(2 k-1)^{2 r+1}} & =\sum_{k=1}^{n} \frac{1}{(2 k-1)^{2 r+1}}+\frac{1}{(2 n+1)^{2 r+1}} \\
& =\left(1-\frac{1}{2^{2 r+1}}\right) \zeta(2 r+1)+\frac{1}{2^{2 r+1}(2 r) !} \psi^{(2 r)}\left(n+\frac{1}{2}\right)+\frac{1}{(2 n+1)^{2 r+1}} \\
& =\left(1-\frac{1}{2^{2 r+1}}\right) \zeta(2 r+1)+\frac{1}{2^{2 r+1}(2 r) !}\left\{\psi^{(2 r)}\left(n+\frac{1}{2}\right)+\frac{(2 r) !}{\left(n+\frac{1}{2}\right)^{2 r+1}}\right\} \\
& =\left(1-\frac{1}{2^{2 r+1}}\right) \zeta(2 r+1)+\frac{1}{2^{2 r+1}(2 r) !} \psi^{(2 r)}\left(n+\frac{3}{2}\right),
\end{aligned}
$$

which is equal to the right-hand side of (2.6) replaced $n$ by $n+1$. Therefore, by the principle of mathematical induction, (2.6) holds true for all $n \in \mathbb{N}$.

We prove certain inequalities for $\zeta(2 r+1)$ affirmed by Theorem 3 .

Theorem 3 Let $r, n \in \mathbb{N}$ and $N \in \mathbb{N}_{0}$. Then the following inequalities hold true:

$$
\begin{aligned}
& \frac{1}{\left(2^{2 r+1}-1\right)(2 r) !}\left(\frac{(2 r-1) !}{n^{2 r}}+\sum_{k=1}^{2 N+1} \frac{B_{2 k}(1 / 2)}{(2 k) !} \frac{(2 r+2 k-1) !}{n^{2 r+2 k}}\right) \\
& <\zeta(2 r+1)-\frac{2^{2 r+1}}{2^{2 r+1}-1} \sum_{k=1}^{n} \frac{1}{(2 k-1)^{2 r+1}} \\
& <\frac{1}{\left(2^{2 r+1}-1\right)(2 r) !}\left(\frac{(2 r-1) !}{n^{2 r}}+\sum_{k=1}^{2 N} \frac{B_{2 k}(1 / 2)}{(2 k) !} \frac{(2 r+2 k-1) !}{n^{2 r+2 k}}\right) .
\end{aligned}
$$

Proof It is known [18] that, for $x>\frac{1}{2}$ and $N \in \mathbb{N}_{0}$,

$$
\begin{aligned}
& \frac{(n-1) !}{\left(x-\frac{1}{2}\right)^{n}}+\sum_{k=1}^{2 N+1} \frac{B_{2 k}(1 / 2)}{(2 k) !} \frac{(n+2 k-1) !}{\left(x-\frac{1}{2}\right)^{n+2 k}} \\
& <(-1)^{n+1} \psi^{(n)}(x) \\
& <\frac{(n-1) !}{\left(x-\frac{1}{2}\right)^{n}}+\sum_{k=1}^{2 N} \frac{B_{2 k}(1 / 2)}{(2 k) !} \frac{(n+2 k-1) !}{\left(x-\frac{1}{2}\right)^{n+2 k}} \quad(n \in \mathbb{N}),
\end{aligned}
$$


where

$$
B_{k}(1 / 2)=-\left(1-\frac{1}{2^{k-1}}\right) B_{k} \quad\left(k \in \mathbb{N}_{0}\right)
$$

and $B_{k}$ are Bernoulli numbers.

By replacing $x$ and $n$ in (2.8) by $n+\frac{1}{2}$ and $2 r$, respectively, we get

$$
\begin{aligned}
& \frac{(2 r-1) !}{n^{2 r}}+\sum_{k=1}^{2 N+1} \frac{B_{2 k}(1 / 2)}{(2 k) !} \frac{(2 r+2 k-1) !}{n^{2 r+2 k}} \\
& <-\psi^{(2 r)}\left(n+\frac{1}{2}\right) \\
& <\frac{(2 r-1) !}{n^{2 r}}+\sum_{k=1}^{2 N} \frac{B_{2 k}(1 / 2)}{(2 k) !} \frac{(2 r+2 k-1) !}{n^{2 r+2 k}} \quad\left(r \in \mathbb{N} ; N \in \mathbb{N}_{0}\right) .
\end{aligned}
$$

Write (2.6) as

$$
\begin{aligned}
& \zeta(2 r+1)-\frac{2^{2 r+1}}{2^{2 r+1}-1} \sum_{k=1}^{n} \frac{1}{(2 k-1)^{2 r+1}} \\
& =\frac{1}{\left(2^{2 r+1}-1\right)(2 r) !}\left(-\psi^{(2 r)}\left(n+\frac{1}{2}\right)\right) \quad(n \in \mathbb{N}) .
\end{aligned}
$$

Upon substituting from (2.9) into (2.10), we obtain the desired result.

\section{Further observations and remarks}

By applying the relation (1.6) to (2.1), we obtain an interesting relationship between the Hurwitz zeta functions $\zeta(2 r, \cdot)$ and $\zeta(2 r+1, \cdot)$ :

$$
\zeta(2 r)-\zeta(2 r, n+2)=\sum_{j=0}^{n} \zeta(2 r+1, j+1)-(n+1) \zeta(2 r+1, n+2) \quad(n, r \in \mathbb{N}) .
$$

Observing, for $r \in \mathbb{N}$,

$$
\zeta(2 r, n+2)=O\left(\frac{1}{n}\right) \quad \text { and } \quad \zeta(2 r+1, n+2)=O\left(\frac{1}{(n+1)^{2}}\right) \quad(n \rightarrow \infty)
$$

and taking the limit on each side of (3.1) as $n \rightarrow \infty$, we also get (2.4).

The formula (2.4) in Theorem 2 can be rewritten in the following double sum:

$$
\zeta(2 r)=\sum_{j=0}^{\infty} \sum_{k=0}^{\infty} \frac{1}{(j+k+1)^{2 r+1}} \quad(r \in \mathbb{N}) .
$$

Inspired by (3.2), we have a natural question: Can the following triple sum:

$$
\sum_{j=0}^{\infty} \sum_{k=0}^{\infty} \sum_{\ell=0}^{\infty} \frac{1}{(j+k+\ell+1)^{2 r+1}} \quad(r \in \mathbb{N})
$$


be expressed in terms of the Hurwitz zeta functions $\zeta(s, a)$ ? Mathematica 8 gives a positive response to this question as well as (3.2):

$$
\sum_{j=0}^{\infty} \sum_{k=0}^{\infty} \sum_{\ell=0}^{\infty} \frac{1}{(j+k+\ell+1)^{2 r+1}}=\frac{1}{2}\{\zeta(2 r)+\zeta(2 r-1)\} \quad(r \in \mathbb{N}) .
$$

However, Mathematica 8 gives no response to the same question for multiple sums more than triple one as in (3.2) and (3.4). In fact, we find that this question for multiple sums more than triple one has already been generally solved as follows (see, e.g., [19, 20], [1, pp.85-86] and [2, pp.151-153]): The n-ple (or, simply, the multiple) Hurwitz zeta function $\zeta_{n}(s, a)$ is defined by

$$
\zeta_{n}(s, a):=\sum_{k_{1}, \ldots, k_{n}=0}^{\infty}\left(a+k_{1}+\cdots+k_{n}\right)^{-s} \quad\left(\Re(s)>n ; n \in \mathbb{N} ; a \in \mathbb{C} \backslash \mathbb{Z}_{0}^{-}\right) .
$$

Choi [19] (see also Choi and Srivastava [20]) found that $\zeta_{n}(s, a)$ is expressible as the following finite linear combination of the generalized zeta functions $\zeta(s, a)$ with polynomial coefficients in $a$ :

$$
\zeta_{n}(s, a)=\sum_{j=0}^{n-1} p_{n, j}(a) \zeta(s-j, a)
$$

where

$$
p_{n, j}(a):=\frac{1}{(n-1) !} \sum_{\ell=j}^{n-1}(-1)^{n+1-j}\left(\begin{array}{l}
\ell \\
j
\end{array}\right) s(n, \ell+1) a^{\ell-j} .
$$

Here $s(n, \ell)$ denotes Stirling numbers of the first kind which satisfy the following recurrence relations:

$$
s(n+1, \ell)=s(n, \ell-1)-n s(n, \ell) \quad(n \geqq \ell \geqq 1)
$$

and

$$
\left(\begin{array}{l}
k \\
j
\end{array}\right) s(n, k)=\sum_{\ell=k-j}^{n-j}\left(\begin{array}{l}
n \\
\ell
\end{array}\right) s(n-\ell, j) s(\ell, k-j) \quad(n \geqq k \geqq j) .
$$

It is not difficult to see also that

$$
\begin{aligned}
& s(n, 0)=\left\{\begin{array}{ll}
1 & (n=0), \\
0 & (n \in \mathbb{N}),
\end{array} \quad s(n, n)=1,\right. \\
& s(n, 1)=(-1)^{n+1}(n-1) ! \quad \text { and } \quad s(n, n-1)=-\left(\begin{array}{l}
n \\
2
\end{array}\right) .
\end{aligned}
$$


The series for $\zeta_{n}(s, a)$ can be expressed in terms of the Hurwitz zeta functions $\zeta(s, a)$ explicitly for the first few values of $n$ :

$$
\begin{aligned}
\zeta_{2}(s, a)= & (1-a) \zeta(s, a)+\zeta(s-1, a) ; \\
\zeta_{3}(s, a)= & \frac{1}{2}\left(a^{2}-3 a+2\right) \zeta(s, a)+\left(\frac{3}{2}-a\right) \zeta(s-1, a)+\frac{1}{2} \zeta(s-2, a) ; \\
\zeta_{4}(s, a)= & \frac{1}{6}\left\{\left(-a^{3}+6 a^{2}-11 a+6\right) \zeta(s, a)+\left(3 a^{2}-12 a+11\right) \zeta(s-1, a)\right. \\
& -(3 a-6) \zeta(s-2, a)+\zeta(s-3, a)\} .
\end{aligned}
$$

We find from (1.3) that the special cases $(s=2 r+1$ and $a=1)$ of the first and second equations in (3.11) yield immediately (3.2) and (3.4), respectively.

In view of (1.14) and (2.4), we get a closed-form evaluation of series involving the zeta functions as follows:

$$
\sum_{j=0}^{\infty} \zeta(2 r+1, j+1)=(-1)^{r+1} \frac{(2 \pi)^{2 r}}{2(2 r) !} B_{2 r} \quad(r \in \mathbb{N}) .
$$

An interesting historical introduction to the remarkably widely and extensively investigated subject of closed-form evaluation of series involving the zeta functions was presented (see [1] and [2]). A considerably large number of formulas have been derived, by using various methods and techniques, in the vast literature on this subject (see, e.g., [1, Chapter 3], [2, Chapter 3] and references therein).

It is noted that, in fact, the formula (2.6) is seen to be easily deduced from the following known relation (cf., (1.8)):

$$
\zeta(s, a)=\zeta(s, n+a)+\sum_{k=1}^{n} \frac{1}{(k+a-1)^{s}} \quad(n \in \mathbb{N})
$$

and the relation in (1.6). But we record it in the form of a lemma in order to use it in the proof of Theorem 3.

The special case $(N=0)$ of $(2.7)$ becomes

$$
\begin{aligned}
& \frac{1}{\left(2^{2 r+1}-1\right)(2 r) !}\left(\frac{(2 r-1) !}{n^{2 r}}-\frac{(2 r+1) !}{24 n^{2 r+2}}\right) \\
& <\zeta(2 r+1)-\frac{2^{2 r+1}}{2^{2 r+1}-1} \sum_{k=1}^{n} \frac{1}{(2 k-1)^{2 r+1}}<\frac{1}{\left(2^{2 r+1}-1\right)(2 r) !}\left(\frac{(2 r-1) !}{n^{2 r}}\right) \\
& \quad(r, n \in \mathbb{N})
\end{aligned}
$$

whose further special case when $r=1$ is

$$
\frac{1}{14 n^{2}}-\frac{1}{56 n^{4}}<\zeta(3)-\frac{8}{7} \sum_{k=1}^{n} \frac{1}{(2 k-1)^{3}}<\frac{1}{14 n^{2}} \quad(n \in \mathbb{N}) .
$$


The special case $(N=1)$ of $(2.7)$ yields

$$
\begin{aligned}
& \frac{1}{\left(2^{2 r+1}-1\right)(2 r) !}\left(\frac{(2 r-1) !}{n^{2 r}}-\frac{(2 r+1) !}{24 n^{2 r+2}}+\frac{7(2 r+3) !}{5,760 n^{2 r+4}}-\frac{31(2 r+5) !}{967,680 n^{2 r+6}}\right) \\
& <\zeta(2 r+1)-\frac{2^{2 r+1}}{2^{2 r+1}-1} \sum_{k=1}^{n} \frac{1}{(2 k-1)^{2 r+1}} \\
& <\frac{1}{\left(2^{2 r+1}-1\right)(2 r) !}\left(\frac{(2 r-1) !}{n^{2 r}}-\frac{(2 r+1) !}{24 n^{2 r+2}}+\frac{7(2 r+3) !}{5,760 n^{2 r+4}}\right) \quad(r, n \in \mathbb{N}) .
\end{aligned}
$$

A further special case $(r=1)$ of $(3.16)$ is

$$
\begin{aligned}
& \frac{1}{14}\left(\frac{1}{n^{2}}-\frac{1}{4 n^{4}}+\frac{7}{48 n^{6}}-\frac{31}{192 n^{8}}\right) \\
& \quad<\zeta(3)-\frac{8}{7} \sum_{k=1}^{n} \frac{1}{(2 k-1)^{3}}<\frac{1}{14}\left(\frac{1}{n^{2}}-\frac{1}{4 n^{4}}+\frac{7}{48 n^{6}}\right) \quad(n \in \mathbb{N}) .
\end{aligned}
$$

\section{Competing interests}

The authors declare that they have no competing interests.

\section{Authors' contributions}

The authors have equal contributions to each part of this paper. All authors have read and approved the final manuscript

\section{Author details}

${ }^{1}$ Department of Mathematics, Dongguk University, Gyeongju, 780-714, Republic of Korea. ${ }^{2}$ School of Mathematics and Informatics, Henan Polytechnic University, Jiaozuo City, Henan Province 454003, People's Republic of China.

\section{Acknowledgements}

The first author is supported by the Basic Science Research Program through the National Research Foundation of Korea funded by the Ministry of Education, Science and Technology (2012-0002957)

Received: 4 September 2012 Accepted: 9 January 2013 Published: 28 February 2013

\section{References}

1. Srivastava, HM, Choi, J: Series Associated with the Zeta and Related Functions. Kluwer Academic, Dordrecht (2001)

2. Srivastava, HM, Choi, J: Zeta and q-Zeta Functions and Associated Series and Integrals. Elsevier, Amsterdam (2012)

3. Riemann, B: Über die Anzahl der Primzahlen unter einer gegebenen Grösse. Monatsber. Akad. Berlin 1859, 671-680 (1859)

4. Edwards, HM: Riemann's Zeta Function. Academic Press, New York (1974); Dover Publications, Mineola, New York (2001)

5. Devlin, K: The Millennium Problems. Basic Books, New York (2002)

6. Pengelley, DJ: Dances between continuous and discrete: Euler's summation formula. In: Bradley, R, Sandifer, E (eds.) Proceedings, Euler $2 k+2$ Conference (Rumford, Maine, 2002) (2003)

7. Tsumura, H: An elementary proof of Euler's formula for $\zeta(2 m)$. Am. Math. Mon. 111, 430-431 (2004)

8. Apéry, R: Irrationalité de $\zeta(2)$ et $\zeta(3)$. In: Journées Arithmétiques de Luminy (Colloq. Internat. CNRS, Centre Univ. Luminy, Luminy, 1978), pp. 11-13, Astérisque 61. Soc. Math. France, Paris (1979)

9. Rivoal, T: La fonction zêta de Riemann prend une infinité de valeurs irrationnelles aux entiers impairs. C. R. Acad. Sci. Paris 331, Série I 267-270 (2000)

10. Zudilin, W: One of the numbers $\zeta(5), \zeta(7), \zeta(9), \zeta(11)$ is irrational. Russ. Math. Surv. 56, 774-776 (2001)

11. Zhang, N-Y: Ramanujan's formulas and the values of the Riemann zeta function at odd positive integers. Adv. Math. 12, 61-71 (1983) (Chinese)

12. Hardy, GH: A formula of Ramanujan. J. Lond. Math. Soc. 3, 238-240 (1928)

13. Grosswald, E: Comments on some formulae of Ramnujan. Acta Arith. 21, 25-34 (1972)

14. Grosswald, E: Die Werte der Riemannschen Zeta Function an ungeraden Argumenstellen. Gött. Nachr. 1970, 9-13 (1970)

15. Dancs, MJ, He, T-X: An Euler-type formula for $\zeta(2 k+1)$. J. Number Theory $118,192-199(2006)$

16. Chen, C-P: Inequalities and series formula for $\zeta(3)$. Preprint

17. Abramowitz, M, Stegun, IA (eds.): Handbook of Mathematical Functions with Formulas, Graphs, and Mathematical Tables, 10th printing, National Bureau of Standards, Applied Mathematics Series, vol. 55. National Bureau of Standards, Washington (1972); Reprinted by Dover Publications, New York (1965)

18. Allasia, G, Giordano, C, Pećarić, J: Inequalities for the gamma function relating to asymptotic expansions. Math. Inequal. Appl. 5(3), 543-555 (2002) 
19. Choi, J: Explicit formulas for the Bernoulli polynomials of order $n$. Indian J. Pure Appl. Math. 27, 667-674 (1996)

20. Choi, J, Srivastava, HM: The multiple Hurwitz Zeta function and the multiple Hurwitz-Euler eta function. Taiwan. J. Math. 15, 501-522 (2011)

doi:10.1186/1029-242X-2013-75

Cite this article as: Choi and Chen: Certain relationships among polygamma functions, Riemann zeta function and generalized zeta function. Journal of Inequalities and Applications 2013 2013:75.

Submit your manuscript to a SpringerOpen ${ }^{\circ}$ journal and benefit from:

- Convenient online submission

- Rigorous peer review

- Immediate publication on acceptance

Open access: articles freely available online

- High visibility within the field

- Retaining the copyright to your article

Submit your next manuscript at $>$ springeropen.com 